\title{
An Improved Modeling of Nonlinear Circuits based on Elman Neural Network
}

\author{
Shao JIE ${ }^{1,2, *}$, Shu JIAN ${ }^{1,2}$, Wang LI ${ }^{1,2}$ and Reza Malekian ${ }^{3, *}$ \\ ${ }^{1}$ Key Laboratory of Radar Imaging and Microwave Photonics (Nanjing Univ. Aeronaut. Astronaut.), Ministry of Education, College of \\ Electronic and Information Engineering, Nanjing University of Aeronautics and Astronautics, Nanjing, 210016, China \\ ${ }^{2}$ Key Laboratory of Underwater Acoustic Signal Processing, Ministry of Education, Southeast University, Nanjing, 210096, China \\ ${ }^{3}$ Department of Electrical, Electronic and Computer Engineering, University of Pretoria, Pretoria, 0002, South Africa
}

Received: 25 Jul. 2013, Revised: 27 Oct. 2013, Accepted: 28 Oct. 2013

Published online: 1 Jul. 2014

\begin{abstract}
In this paper, an improved modeling based on Elman neural network was proposed to analyze the nonlinear features of nonlinear circuits with the memory effect. The input vector of the hidden layer in neural network is normalized to enhance the neural network convergence precision. A group of Chebyshev orthogonal basis functions was employed to activate hidden layer neurons. Computer simulation results of the nonlinear power amplifier (PA) have shown that the proposed behavioral modeling not only accurately describes the nonlinear distortions of PAs, but also well depicts memory effect of PAs. And, the proposed approach could be also applied to analyze linear circuits and RF power amplifiers.
\end{abstract}

Keywords: Nonlinear circuits, Behavioral Model, Elman Neural Network, Chebyshev Orthogonal Basis Functions

\section{Introduction}

As a kind of nonlinear circuit, The output transistors of Class-D power amplifiers (CDPAs) operate in the ohmic and cut-off regions, therefore, the CDPAs nonlinearity [1], including the harmonic distortion and the intermodulation distortion (IMB) $[2,3]$, is very serious. This switching-mode PA can also provide high efficiency at RF and microwave frequencies.

The behavioral modeling [4] is an effective way to study PA's nonlinear characteristics. Currently, the main PA's behavioral modeling with the memory effect is Volterra series method [5]. Since many of the coefficients in Volterra series model, it is difficult to use in practical applications. Thus, there have been various improved PA's models based on Volterra series.

Because the neural networks (NNs) can be provided with a good approximation for nonlinear functions and systems, the modeling methods of PA based on NNs are being taken seriously [6-8]. In order to research the distortion behavior of nonlinear PAs, an improved modeling based on Elman neural network (ENN) was proposed in this paper. A group of Chebyshev orthogonal basis functions [9] instead of sigmoid functions were employed as the activation functions of hidden layer. The gradient descent algorithm was derived to train the connecting weights of improved ENN to enhance learning ability of model and raise its convergence speed. Numerical simulation results have shown that the improved model could well describes the nonlinear features of PA circuits.

\section{The Basic Elman Neural Network}

The architecture of basic Elman neural network (BENN) [6] is illustrated in Fig. 1. The BENN model is composed of four layers. The input layer $u_{r}(k),(r=1,2, \ldots, R)$, has $R$ nodes. It accepts the input variables and transmits to the hidden layer. The hidden layer $F_{j}(k),(j=1,2, \ldots, L)$, has $L$ nodes and contains the transfer function $f(p)$. The context layer $x_{l}^{c}(k),(l=1,2, \ldots, L)$ is the feedback loop of hidden layer and it has $L$ neural nodes, too. The output layer has $M$ nodes and the output $y_{m}(k),(m=1,2, \ldots, M)$, is the linear combination of the hidden layer output.

$$
\begin{gathered}
y(k)=W^{3}(k) F(k) \\
F(k)=f[P(k)]
\end{gathered}
$$

\footnotetext{
*Corresponding author e-mail: shaojie@ nuaa.edu.cn, reza.malekian@iee.org
} 


$$
\begin{gathered}
P(k)=W^{2}(k) u+W^{1}(k) X^{c}(k) \\
X^{c}(k)=F(k-1)
\end{gathered}
$$

Where $W^{3}(k)$ is the $L \times M$ dimensional weight matrix from the hidden layer to the output layer. $W^{2}(k)$ is the $R \times L$ dimensional weight matrix from the input layer to the hidden layer. $W^{1}(k)$ is the $L \times L$ dimensional weight matrix from the context layer to the hidden layer. $k$ is the number of the iteration steps and $f(p)$ usually represents the sigmoid function.

Because training and convergence speed of BENN [10] are usually very slow and it is not suitable for time critical applications, some improved models base on Elman neural network have been proposed recently and certificated to have some advantages over the BENN $[11,12]$.

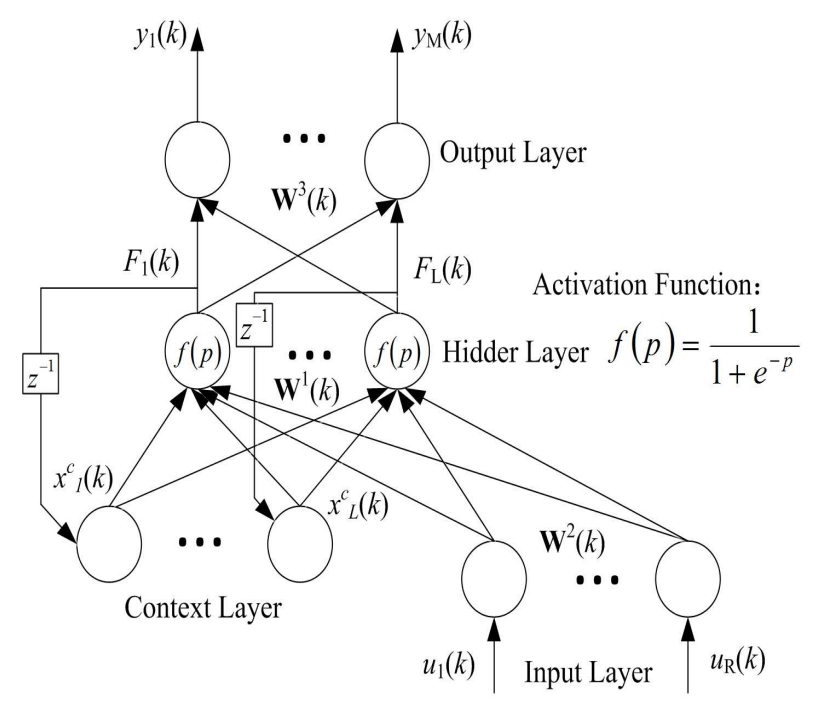

Fig. 1: The architecture of BENN

\section{The Improved Model Based on ENN}

\subsection{The Chebyshev orthogonal basis function}

According to the Chebyshev recursive formula, the Chebyshev orthogonal polynomials are expressed as

$$
\begin{cases}f_{1}(p)=1 & \\ f_{2}(p)=p & 1 \leq j \leq L-2 \\ \cdot & \\ \cdot & \\ f_{j+2}(p)=2 p f_{j+1}(p)-f_{j}(p) & \end{cases}
$$

The Chebyshev functions are used to Chebyshev Functional Neural Network in [9]. To raise learning speed and enhance convergence precision of behavioral model of PAs, a group of Chebyshev orthogonal polynomial functions were used to activate the hidden layer neurons instead of sigmoid functions in Elman neural network.

The input of Chebyshev orthogonal basis functions [13] is defined within the interval $[-1,1]$. Therefore, the input vector $P(k)$ has to be appropriately normalized.

$$
\begin{gathered}
\bar{P}(k)=P(k) / \max [\operatorname{abs}(P(k))] \\
F(k)=f[\bar{P}(k)]
\end{gathered}
$$

Where $\max [\operatorname{abs}(\mathbf{P}(k))]$ is the maximum absolute unit of the input vector in hidden layer. $\bar{P}(k)$ is the normalized input vector of hidden layer.

\subsection{Training Algorithm}

In improved ENN, a supervised learning is used to train the connecting weights of improved ENN based on the gradient descent algorithm. The BP algorithm is applied to adjust parameters of improved ENN by using the training patterns. By recursive algorithm of the chain rule, the iteration relationships of connecting weights of improved ENN to the corresponding layers are then given.

Assume that the desired system output vector is $\tilde{y}(k)=\left[\tilde{y_{1}}(k), \tilde{y_{2}}(k), \ldots, \tilde{y_{M}}(k)\right]^{T}$ and the proposed model output vector is $y(k)=\left[y_{1}(k), y_{2}(k), \ldots, y_{M}(k)\right]^{T}$. The error function $E(k)$ [10] can be defined as:

$$
E(k)=\frac{1}{2}[\tilde{y}(k)-y(k)]^{T}[\tilde{y}(k)-y(k)]
$$

Demonstrate the partial derivatives of the connecting weights $W^{3}(k), W^{2}(k)$, and $W^{1}(k)$ by means of the error function $E(k)$, respectively. The improved ENN's training algorithm is expressed by the gradient descent algorithm [2].

$$
\Delta w_{j l}^{1}(k)=\eta_{1} \sum_{m=1}^{M}\left[\delta_{m}(k) w_{m j}^{3}(k)\right] \partial F_{j}(k) / \partial w_{j l}^{1}(k)
$$

$$
\Delta w_{j r}^{2}(k)=\eta_{2} \lambda_{j}(k) u_{r}(k)
$$

$$
\Delta w_{m j}^{3}(k)=\eta_{3} \delta_{m}(k) F_{j}(k)
$$

with

$$
\delta_{m}(k)=\tilde{y}_{m}(k)-y_{m}(k)
$$

$\lambda_{j}(k)=\sum_{m=1}^{M}\left[\delta_{m}(k) w_{m j}^{3}(k)\right] f_{j}^{\prime}(.) / \max _{1 \leq j \leq L}\left(\left|p_{j}(k)\right|\right)$ 


$$
\frac{\partial F_{j}(k)}{\partial w_{j l}^{1}(k)}=f_{j}^{\prime}(.) x_{l}^{c}(k-1) / \max _{1 \leq j \leq L}\left(\left|p_{j}(k)\right|\right)
$$

It has to be noticed that $f_{j}^{\prime}($.$) in equation (13), (14)$ denote the first derivative of the first category Chebyshev orthogonal basis functions. $\eta_{1}, \eta_{2}$ and $\eta_{3}$ represent the learning rate of the connecting weights.

In order to well analyze the error of system output and improved ENN output, the time domain error function $e(k)$ is termed as:

$$
e(k)=|y(k)-\tilde{y}(k)|
$$

The basic steps to determine the required optimal number of neurons in hidden layer are provided as follows.

Step1: Initialize iteration step $k=0$, the weights $W^{3}(k), W^{2}(k)$ and $W^{1}(k)$ of improved ENN are as 0 , and corresponding learning rates are $\eta_{1}=0.05$ , $\eta_{2}=\eta_{3}=0.004$. Set initialized number of neurons in hidden layer $n=4$, define maximum neurons in hidden layer $n_{\max }=50$, maximum iteration steps $N=100$, threshold value of the error $\varepsilon_{\min }=-30 \mathrm{~dB}$.

Step2: Increase the number of iteration step $k=k+1$; if $k>N$,end the training process.

Step3: Normalize the vector $P(k)$ in hidden layer;

Step4: According to formula (1), (7) and (4), calculate the outputs of every neuron in every layer and the error function $E(k)$ of $k$ th iteration step. If $E(k)$ is less than $\varepsilon_{\text {min }}$, end the training process, else execute step 5.

Step5: Acquire the adjustment values of the weight matrixes according to formulas (9) to (11), Update the weights $w_{j l}^{1}(k+1)=w_{j l}^{1}(k)+\Delta w_{j l}^{1}(k), w_{j r}^{2}(k+1)=$ $w_{j r}^{2}(k)+\Delta w_{j r}^{2}(k)$ and $w_{i j}^{3}(k+1)=w_{i j}^{3}(k)+\Delta w_{i j}^{3}(k)$. Jump to step 2.

Step6: Increase the number of hidden layer neurons $n$, if $n>n_{\max }$, end the training process. Jump to step2.

\section{Simulation results and analysis}

In order to verify the correctness and reliability of improved ENN model, the training sample sequence is achieved from the two-tone input of half-bridge CDPA shown in Fig. 2.

As shown in Fig. 2, the PWM signal $q$ is produced by two-tone signal and triangular signal, and its output logic level is $\pm 12 \mathrm{~V}$. The frequencies of two-tone signal are $f_{1}=3 \mathrm{kHz}$ and $f_{2}=430 \mathrm{~Hz}$, and the amplitudes are $A M_{1}=A M_{2}=4 \mathrm{~V}$. The frequency and amplitude of triangular signal are determined as $f_{3}=40 \mathrm{kHz}$ and $A M_{3}=9.6 \mathrm{~V}$. A group of the training samples $\tilde{y}$ are extracted from $10 \mathrm{~ms}$ to $11 \mathrm{~ms}$ by the sampling frequency $f_{s}=100 \mathrm{kHz}$.

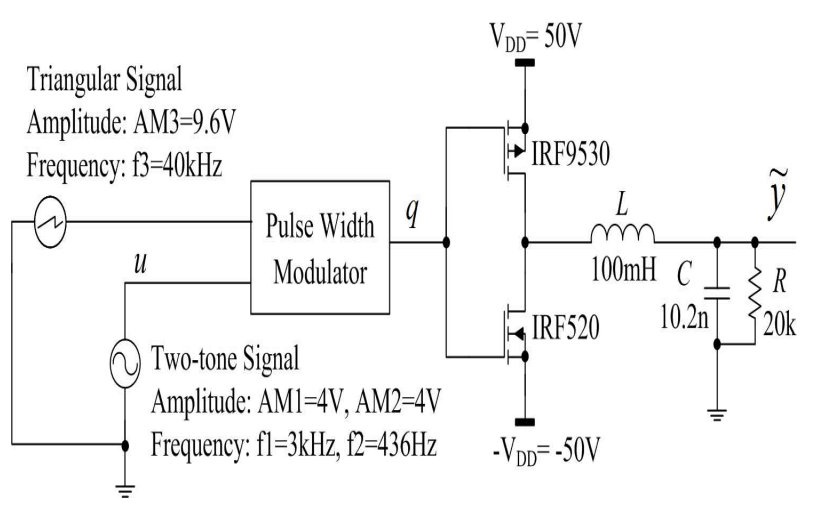

Fig. 2: The circuit of half-bridge CDPA
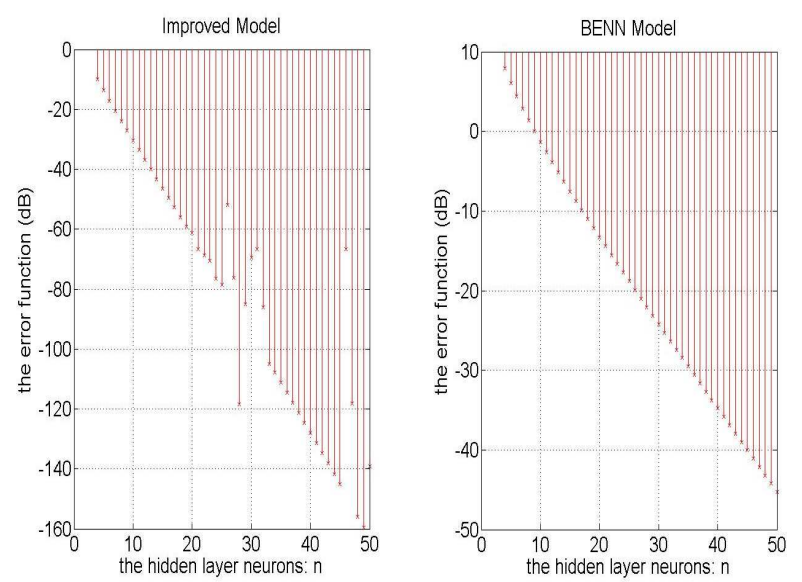

Fig. 3: The error functions versus the hidden layer neurons, left:Improved model, right: BENN model

\subsection{Optimal Neurons Number in Hidden Layer}

In order to determine the optimal neurons number in hidden layer of two models, the relationship between the error function $E(k)$ and the hidden layer neurons $n$ is presented, as shown in Fig. 3.

It can be seen in Fig. 3 that the error $E(k)$ of BENN model is $-1.33 \mathrm{~dB}$, The error $E(k)$ of improved ENN model is only $-27.96 \mathrm{~dB}$ under the same conditions $(\mathrm{N}=100, \mathrm{n}=10)$. The improved ENN model's convergence speed and stability is superior than BENN model, distinctly, although the error $E(k)$ of both models declines rapidly with the increase of the number of hidden layer neurons when iteration steps $N=100$.

If the threshold value of the error function is $\varepsilon_{m} i n=$ $-28 d B$, the optimal number of neurons in hidden layer of proposed model can be determined as $n=10$ by analyzing Fig. 3. 

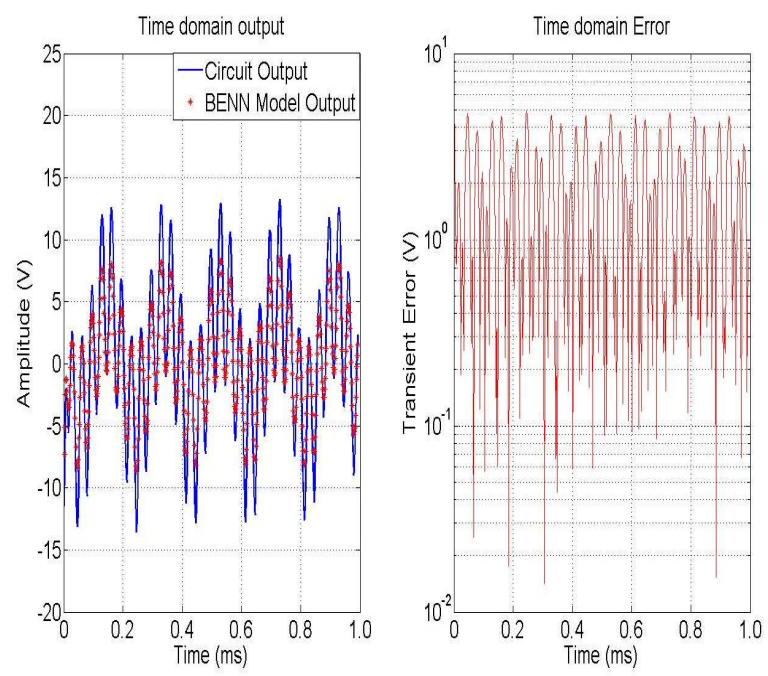

Fig. 4: Comparison between circuit output and BENN model in time domain
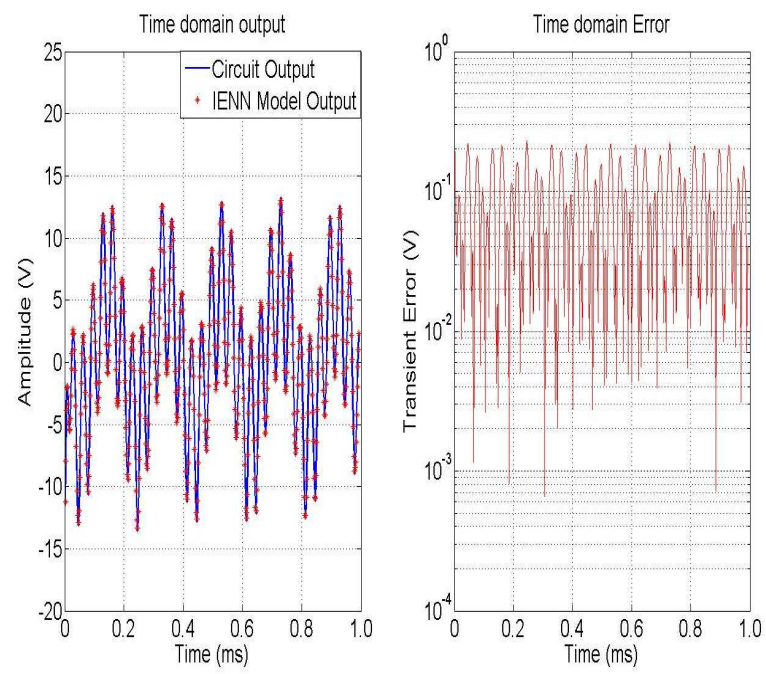

Fig. 5: Comparison between circuit output and improved ENN model in time domain

\subsection{Simulation Analysis of Two Models}

Select the hidden layer neurons $n=10$. The simulation results of half-bridge CDPA base on BENN and improved ENN model are shown in Fig. 4 and Fig. 5 when iteration steps $N=100$. In different number of hidden layer neurons, the error $E(k)$ and the maximum time domain error $e_{\max }$ of two models are listed in Table 1. Similarly, the error $E(k)$ and the maximum time domain error $e_{\max }$ of two models are presented in Table 2 when the iteration steps $N=200$.
Table 1: $E(k)$ and $e_{\max }$ of two models $(N=100)$

\begin{tabular}{|c|c|c|c|}
\hline model & $\mathrm{n}$ & $\mathrm{E}(\mathrm{k})(\mathrm{dB})$ & $e_{\max }(V)$ \\
\hline BENN model & 7 & 1.33 & $6.7026 \times 10^{0}$ \\
\hline BENN model & 10 & -1.33 & $4.9379 \times 10^{0}$ \\
\hline BENN model & 13 & -3.99 & $3.6314 \times 10^{0}$ \\
\hline proposed model & 7 & -17.21 & $7.9330 \times 10^{-1}$ \\
\hline proposed model & 10 & -27.96 & $2.2910 \times 10^{-1}$ \\
\hline proposed modell & 13 & -38.92 & $6.5100 \times 10^{-2}$ \\
\hline
\end{tabular}

Table 2: $E(k)$ and $e_{\max }$ of two models $(N=200)$

\begin{tabular}{|c|c|c|c|}
\hline model & $\mathrm{n}$ & $\mathrm{E}(\mathrm{k})(\mathrm{dB})$ & $e_{\max }(V)$ \\
\hline BENN model & 7 & -13.25 & $3.3086 \times 10^{0}$ \\
\hline BENN model & 10 & -20.13 & $1.7958 \times 10^{0}$ \\
\hline BENN model & 13 & -26.58 & $9.7120 \times 10^{-1}$ \\
\hline proposed model & 7 & -41.87 & $4.6400 \times 10^{-2}$ \\
\hline proposed model & 10 & -63.45 & $3.9000 \times 10^{-3}$ \\
\hline proposed model & 13 & -85.31 & $3.1225 \times 10^{-4}$ \\
\hline
\end{tabular}

It can be seen in Table 1 and Table 2, with the increase of the iteration steps, both $E(k)$ and $e_{\max }$ decrease. The larger the number of hidden neurons $n$ is, the smaller both $E(k)$ and $e_{\max }$ are. To reach the approximate error $E(k)$ with the same iteration steps, improved ENN needs fewer hidden layer neurons than BENN. When the hidden layer neurons and iteration steps are same, the proposed ENN model has faster convergence speed and higher convergence precision than BENN model.

By the above comparison and analysis, The proposed ENN model is superior to the BENN model in both accuracy and calculation in time domain, which means a significant improvement of performance based on proposed model.

Based on proposed ENN model, the frequency characteristics of simulation results are given in Fig. 6 when $n=10$ and $N=200$. The spectrum error between circuit output and proposed ENN model output is shown as Fig. 7.

The magnitudes and frequencies of IMD products of the half-bridge CDPA are listed in Table 3. Due to the

Table 3: IMD products of the half-bridge CDPA

\begin{tabular}{|l|c|c|c|c|}
\hline IMD prod. & lower IMD2 & upper IMD2 & lower IMD3 & upper IMD3 \\
\hline Frequency & 2570 & 3430 & 2140 & 3860 \\
\hline Magnitude & $-6.84 \mathrm{~dB}$ & $-4.03 \mathrm{~dB}$ & $-2.79 \mathrm{~dB}$ & $2.88 \mathrm{~dB}$ \\
\hline
\end{tabular}

memory effect, the IMD products are asymmetries in lower and upper sidebands [14]. The IMD products are 


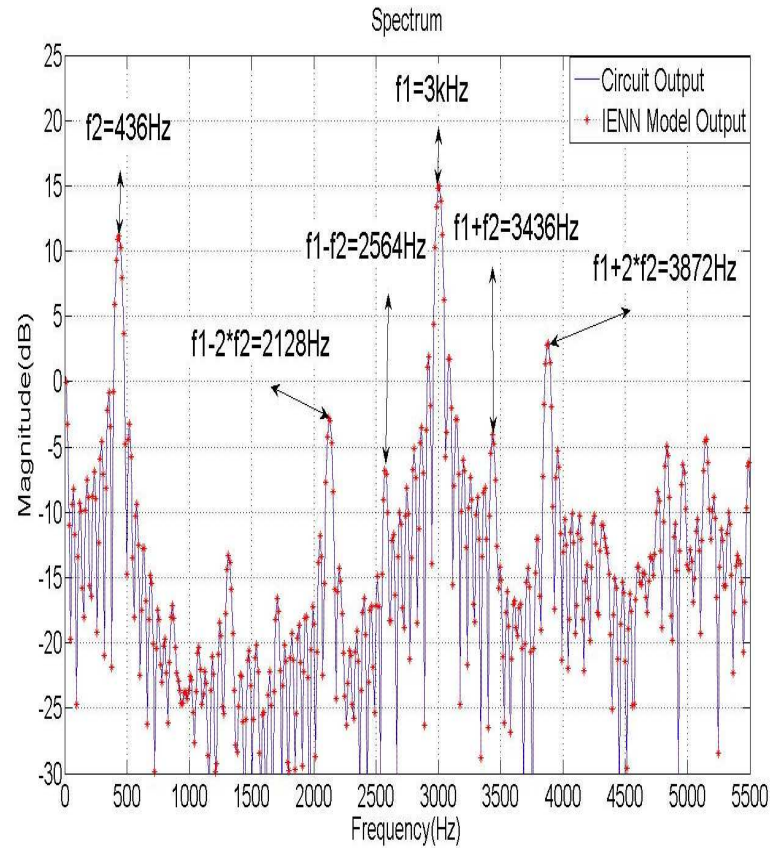

Fig. 6: The spectrum of circuit output and improved model.

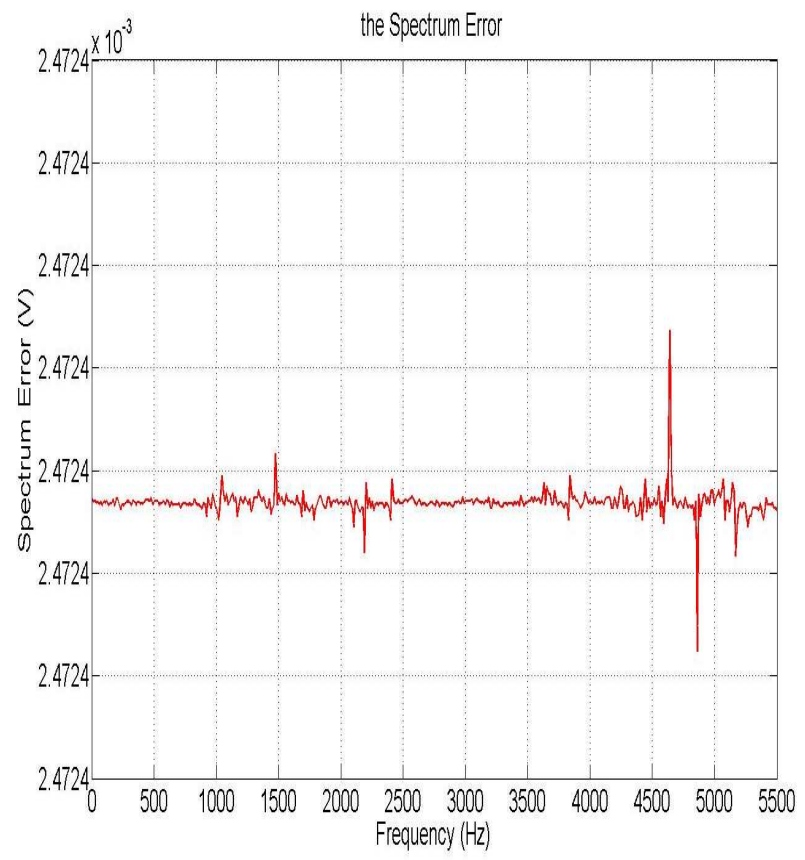

Fig. 7: The spectrum error between circuit output and improved model. asymmetries too in Table 3 , which is caused by the memory effect of CDPAs. It can be known in Fig. 7 that the maximum spectrum error between the circuit output and the proposed ENN model output is less than $2.4724 \times 10^{-3} \mathrm{~V}$.

Simulation results show that the proposed model can well describe the memory effect of CDPAs. The accuracy of the proposed behavioral model is also high in frequency domain.

\section{Conclusion}

In this paper, an improved modeling based on ENN was proposed to describe the nonlinear features of PAs. In proposed ENN model, a group of Chebyshev orthogonal basis functions was employed to activate hidden layer neurons. The input vector of the hidden-layer in neural network is normalized to enhance the neural network convergence precision. Meanwhile, the optimized algorithm, based on the relationship between the error function and the number of hidden layer neurons, is used to determine the required optimal number of neurons in hidden layer. The gradient descent algorithm was derived to train the connecting weights of improved ENN. The computer simulation results have shown that the proposed behavioral modeling has faster learning speed and higher convergence precision than BENN model in time domain and frequency domain. Furthermore, the proposed model can be used to describe the memory effect of PAs, and could also be applied to analyze the nonlinearity of the RF power amplifiers.

\section{Acknowledgement}

This work was supported in part by the Foundation of Key Laboratory of Chinas Education Ministry and A Project Funded by the Priority Academic Program Development of Jiangsu Higher Education Institutions.

\section{References}

[1] Margaliot, M., Weiss G., The Low-Frequency Distortion in D-Class Amplifiers. IEEE Transactions on Circuits and Systems II: Express Briefs, 57, 772-776 (2010).

[2] Jun Y., T. T. Meng, Cox S. M., Wang-Ling G., TimeDomain Analysis of Intermodulation Distortion of ClosedLoop Class-D Amplifiers. IEEE Transactions on Power Electronics, 27, 2453-2461 (2012).

[3] Francois Koeslag, Hendrik D. Mouton, Johan Beukes, Analytical Modeling of the Effect of Nonlinear Switching Transition Curves on Harmonic Distortion in Class D Audio Amplifiers. IEEE Transactions on Power Electronics, 28, 380-389 (2013). 
[4] Landin P. N., Fritzin J., Van Moer W., Isaksson M., Alvandpour A., Modeling and Digital Predistortion of Class-D Outphasing RF Power Amplifiers. IEEE Transactions on Microwave Theory and Techniques, 60, 1907-1915 (2012).

[5] Tehrani A. S., Haiying C., Afsardoost S., Eriksson T., Isaksson M., Fager C., A Comparative Analysis of the Complexity/Accuracy Tradeoff in Power Amplifier Behavioral Models. IEEE Transactions on Microwave Theory and Techniques, 58, 1510-1520 (2010).

[6] Mkadem, F., S. Boumaiza, Physically Inspired Neural Network Model for RF Power Amplifier Behavioral Modeling and Digital Predistortion. IEEE Transactions on Microwave Theory and Techniques, 59, 913-923 (2011).

[7] Jianfeng Z., Jianyi Z., Lei Z., Wei H., Behavioral Modeling of Power Amplifiers with Dynamic Fuzzy Neural Networks. IEEE Microwave and Wireless Components Letters, 20, 528-530 (2010).

[8] Lippmann, R.P., An introduction to computing with neural nets, ASSP Magazine, IEEE, 4, 4-22 (1987).

[9] Mingyu L., Jinting L., Yang J., Wenjiang F., ComplexChebyshev Functional Link Neural Network Behavioral Model for Broadband Wireless Power Amplifiers. IEEE Transactions on Microwave Theory and Techniques, 60, 1979-1989 (2012).

[10] Qing, S., On the Weight Convergence of Elman Networks. IEEE Transactions on Neural Networks, 21, 463-480 (2010).

[11] Whei-Min L., Chih-Ming H., A New Elman Neural Network-Based Control Algorithm for Adjustable-Pitch Variable-Speed Wind-Energy Conversion Systems. IEEE Transactions on Power Electronics, 26, 473-481 (2011).

[12] Faa-Jeng L., Syuan-Yi C., Kuo-Kai S., Robust Dynamic Sliding-Mode Control Using Adaptive RENN for Magnetic Levitation System. IEEE Transactions on Neural Networks, 20, 938-951 (2009).

[13] Haiquan Z., Jiashu Z., Pipelined Chebyshev Functional Link Artificial Recurrent Neural Network for Nonlinear Adaptive Filter. IEEE Transactions on Systems, Man, and Cybernetics, Part B: Cybernetics, 40, 162-172 (2010).

[14] Hyunchul K., Kenney J. S., Behavioral modeling of nonlinear RF power amplifiers considering memory effects. IEEE Transactions on Microwave Theory and Techniques, 51, 2495-2504 (2003).

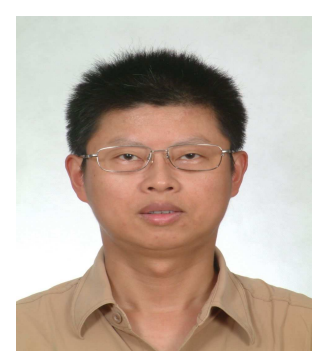

Shao JIE is an associate professor in the the College of Electronic and Information Engineering at Nanjing University of Aeronautics and Astronautics.He is a senior member of Chinese Institute of Electronics.He received the M.S.degree from Department of power engineering, Harbin Engineering University in 1990. His current research interests include digital signal processing and intelligence information processing.

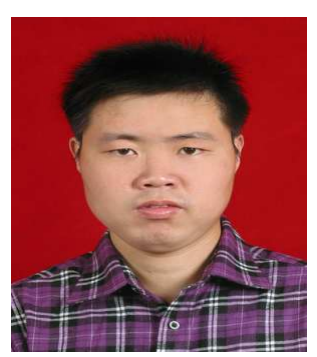

Shu Jian received the M. S. from Nanjing University of Aeronautics and Astronautics China in 2012. He research interests include nonlinear system modeling and signal processing.

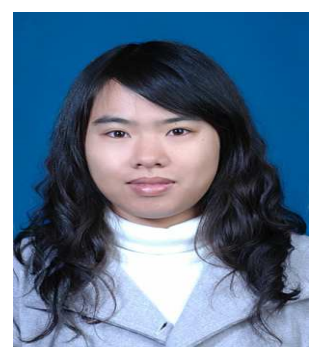

Wang LI received the Bachelor of Engineering degree in communications engineering from Nanjing University of Aeronautics and Astronautics, Jiangsu , China in 2011. She is currently pursuing the Master degree in electronic circuit and system at Nanjing University of Aeronautics and Astronautics. Her research interests include nonlinear system modeling, and feature extraction of signal.

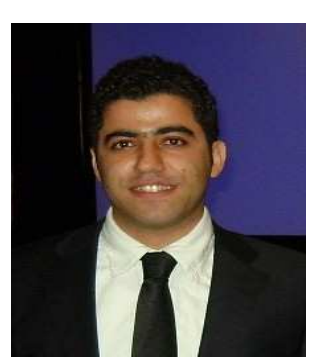

\section{Reza}

Malekian is a Senior Lecturer in the Department of Electrical, Electronic and Computer Engineering at University of Pretoria. Previously, he was an Assistant Professor of Computer Science and Engineering at University for Information Science and Technology St. Paul the Apostle, Republic of Macedonia and a Postdoctoral Fellow in Faculty of Computing, Universiti Teknoligi Malaysia (UTM-Erasmus Partner). He received Ph.D. in Computer Science from UTM, M.Eng. (with honor) in Information Technology Engineering from Iran University of Science and Technology, and B.Eng in Computer Engineering from North University. 